\title{
Quantum quench dynamics of the Luttinger model
}

\author{
A. Iucci ${ }^{1,2,3}$ and M. A. Cazalilla ${ }^{4,3}$ \\ ${ }^{1}$ Instituto de Física la Plata (IFLP)-CONICET and Departamento de Física, Universidad Nacional de La Plata, CC 67, \\ 1900 La Plata, Argentina \\ ${ }^{2}$ DPMC-MaNEP, University of Geneva, 24 Quai Ernest Ansermet, CH-1211 Geneva 4, Switzerland \\ ${ }^{3}$ Donostia International Physics Center (DIPC), Manuel de Lardizábal 4, 20018 San Sebastián, Spain \\ ${ }^{4}$ Centro de Física de Materiales (CFM), Centro Mixto CSIC-UPV/EHU, Edificio Korta, Avenida de Tolosa 72 , \\ 20018 San Sebastián, Spain
}

(Received 18 March 2009; revised manuscript received 22 September 2009; published 8 December 2009)

\begin{abstract}
The dynamics of the Luttinger model after a quantum quench is studied. We compute in detail one- and two-point correlation functions for two types of quenches: from a noninteracting to an interacting Luttinger model and vice versa. In the former case, the noninteracting Fermi gas features in the momentum distribution and other correlation functions are destroyed as time evolves. In the infinite-time limit, equal-time correlations are power laws but the critical exponents are found to differ from their equilibrium values. In all cases, we find that these correlations are well described by a generalized Gibbs ensemble [M. Rigol, V. Dunjko, V. Yurovsky, and M. Olshanii, Phys. Rev. Lett. 98, 050405 (2007)], which assigns a momentum-dependent temperature to each eigenmode.
\end{abstract}

DOI: 10.1103/PhysRevA.80.063619

PACS number(s): 03.75.Ss, 71.10.Pm, 02.30.Ik, 05.70.Ln

\section{INTRODUCTION}

Most of the theoretical efforts in the field of strongly correlated quantum systems over the past few decades have focused on understanding the equilibrium properties of these fascinating systems. For instance, achieving a complete understanding of the phase diagram of rather "simple" models such as the two-dimensional fermionic Hubbard model still remains a huge challenge. Nevertheless, however important these endeavors are, understanding the phase diagram and the equilibrium properties of the phases of strongly correlated systems will not certainly exhaust the possibilities for finding new and surprising phenomena in these complex systems, especially out of equilibrium.

In classical systems, the existence of steady states out of equilibrium is well known. Very often, however, the properties of such states have very little to do with the equilibrium properties of the systems where they occur. Moreover, also very often their existence cannot be inferred from any previous knowledge about the equilibrium phase diagram: they are emergent phenomena.

One good example of a classical nonequilibrium steady state is provided by the appearance of Rayleigh-Bénard convection cells when a fluid layer is driven out of equilibrium by a temperature gradient. Indeed, it is known that dissipation plays an important role in the formation of these classical nonequilibrium states. However, different from classical systems, dissipation in quantum systems causes decoherence, which usually destroys any interesting quantum interference effects. Thus, although one may also wonder if nonequilibrium steady states can also appear when quantum systems are driven out of equilibrium, the study of nonequilibrium phenomena in quantum many-body systems has been regarded, until very recently, as a subject of mostly academic interest. The reason for this may be decoherence due to coupling with the environment, which is always present in most experimental realizations of large quantum many-body sys- tems and which prevents the observation of coherent quantum evolution for long times.

However, the recent availability of highly controllable systems of ultracold atomic gases has finally provided the largely lacking experimental motivation for the study of nonequilibrium phenomena, leading to an explosion of theoretical activity [1-39]. Ultracold atomic gases are especially interesting because they are very weakly coupled to the environment, thus remaining fully quantum coherent for fairly long times (compared to the typical duration of an experiment). At the same time, it is relatively easy to measure the coherent evolution in time of observables such as the density or the momentum distribution. Thus, theorists can now begin to pose questions such as: assuming that a manybody system is prepared in a given initial state that is not an eigenstate of the Hamiltonian, how will it evolve in time? And, more specifically, will it reach a stationary or quasistationary state? If so, what will be the properties of such a state? How much memory will the system retain of its initial conditions?

From another point of view, the problem described in the previous paragraph can be formulated as the study of the response of a system to a sudden perturbation in which the Hamiltonian is changed over a time scale much shorter than any other characteristic time scales of the system. In what follows, we shall refer to this type of experiment as a quantum quench. Quantum quenches are also of particular interest to the "quantum engineering" program for ultracold atomic gases [40]. The reason is that, if we intend to use these highly tunable and controllable systems as quantum simulators of models of many-body physics [such as the twodimensional (2D) fermionic Hubbard model mentioned above], it is utterly important to understand to what extent the final state of the quantum simulator depends on the state in which it was initially prepared. In particular, one is interested in finding out whether the observables in the final state can be obtained from a standard statistical ensemble (say, the microcanonical or the canonical ensemble at an effective 
temperature). If this is so, one would speak of thermalization. If this does not happen, then how much memory does the system retain about its initial state beyond the average energy $E=\langle H\rangle$ ?

We would like to emphasize that the above questions are not merely academic. Indeed, ultracold atomic systems allow for the study of nonadiabatic dynamics when the system is driven between two quantum phases such as a superfluid and a Mott insulator [41,42]. Also, in a recent experiment [43], it has been shown that a faithful realization of the Lieb-Liniger model [44] exhibits absence of thermalization. In other words, when prepared in an nonequilibrium state, the experimental system reached a steady state that cannot be described by any of the "standard" ensembles of statistical mechanics. This absence of thermalization seems to be a consequence of the integrability of the Lieb-Liniger model, that is, the existence of an infinite number of independently conserved quantities. This conclusion was backed by the theoretical analysis of Rigol and co-workers [10], who noticed that the nonequilibrium dynamics of an integrable system is highly constrained. Thus, based on numerical simulations for the Tonks-Girardeau limit of the Lieb-Liniger model, these authors conjectured that the long-time values of some observables should converge to those obtained from a generalized Gibbs ensemble, which can be constructed using the maximum (von Neumann-) entropy principle $[10,45,46]$. The conjecture was first analytically confirmed by analyzing an interaction quench in the Luttinger model by one of us [9]. Later, it has been also found true in other integrable models: Calabrese and Cardy studied a quench in a Harmonic chain [15] and Eckstein and Kollar analyzed the Falikov-Kimball model in infinite dimensions [18] and the $1 / r$ Hubbard model in one dimension [19]. Moeckel and Kehrein [20] studied an interaction quench in the Hubbard model in infinite dimensions by a flow equation method and found that the system reaches an intermediate nonthermal state. Finally, recent numerical studies also have suggested that lack of thermalization may even persist in the absence of integrability in one-dimensional (1D) systems [14] or that it may occur only certain parameter regimes of nonintegrable models [16].

However, it can be expected that [26] for a rather general choice of the initial state, along with a situation where there are few conserved quantities, the system will lose memory of most of the details of the initial state and after it reaches a steady state, the expectation of many experimentally accessible observables such as the particle density or the momentum distribution will look essentially identical to those obtained from a standard thermal ensemble. ${ }^{1}$ Indeed, this is what seems to be observed in the vast majority of the experiments with ultracold atomic gases. However, for experimental many-body systems in general, it is hard to quantify whether this will be always the case. We should take into

\footnotetext{
${ }^{1}$ Implicit in this discussion, it is the fact we are attempting at a description of the system as a whole, not separating its degrees of freedom into a "system" and a "reservoir." We believe that this point of view is more appropriate when discussing ultracold atomic systems, given that they are very weakly coupled to the environment.
}

account that (except perhaps in the case of ultracold atomic gases) the exact form of the quantum Hamiltonian is frequently not known with accuracy. And even when it is known, it is not always possible to tell a priori whether the system is integrable or even if it has other conserved quantities besides the ones assumed by the standard thermodynamic ensembles. As a possible experimental check, we can say that, provided the final result is largely independent of the particular details of the preparation of the initial state and that the observations agree with those obtained from a thermal ensemble, we can say that thermalization has occurred. Indeed, some recent numerical evidences [23], supplemented by the extension to many-particle systems of a conjecture known as "eigenstate thermalization hypothesis" (first introduced in the context of quantum chaos [47]), seem to indicate that lack of integrability will in general lead to thermalization (in the sense defined above). Indeed, Reimann [26] recently analytically demonstrated that, under realistic experimental conditions, equilibration will be observed in an isolated system that has been initially prepared in an nonequilibrium mixed state. Nevertheless, even if the issue of thermalization for nonintegrable systems may have been settled, other questions such as the details of the transition from the integrable case (which thermalizes to a generalized Gibbs ensemble) to the nonintegrable case (which thermalizes to the standard microcanonical or Gibbs ensemble for large enough systems) are questions that are still far from being completely understood. ${ }^{2}$

In this paper, we will not try to answer the difficult questions posed in the previous paragraph. Instead, we focus on analyzing the quench dynamics of a relatively well-known one-dimensional model, namely, the Luttinger model (LM). A brief account of the results in this paper has been already published elsewhere [9]. In a future publication, we shall also deal with another closely related model: the sineGordon model [48].

The LM Hamiltonian can be represented as a quadratic form of creation and destruction operators. The long-time behavior following a quench for Hamiltonians of this form was recently considered by Barthel and Schollwöck [49]. These authors provided some general conditions for the appearance of dephasing and steady nonthermal states. This question was also taken up recently by Kollar and Eckstein [19]. However, since the Luttinger model may be relevant to experiments using ultracold atomic gases (see Sec. IV) or numerical simulations, it is important to obtain analytical results. The simplicity of this model also allows us to test in detail a number of general results $[12,49]$.

The rest of this paper is organized as follows. In Sec. II, we discuss the evolution of some simple correlation functions of the Luttinger model. We consider the case where the interaction between the fermions is suddenly switched on and the reverse situation, that is, when the interaction in suddenly switched off. In Sec. III, we discuss how the infinite-

\footnotetext{
${ }^{2}$ This question is also related to the problems concerning the applicability of the maximum entropy approach $[45,46]$ to statistical mechanics. See for instance the critique by Ma in Ref. [64]. However, the maximum entropy approach is advocated by Balian [59].
} 
time behavior of some of the correlation functions following a quantum quench can be obtained from a generalized Gibbs ensemble. We also discuss some observables for which this conjecture fails. The experimental relevance of our results is briefly discussed in Sec. IV, along with other conclusions of this work. Finally, the details of some of the lengthier calculations are provided in Appendixes A to C.

\section{LUTTINGER MODEL}

The LM describes a 1D system of interacting fermions with linear dispersion. It was first introduced by Luttinger [50] but its complete solution was only later obtained by Mattis and Lieb [51], who showed that the elementary excitations of the system are not fermionic quasiparticles. Instead, Mattis and Lieb introduced a set of bosonic fields describing collective density modes (phonons) of the system, which are the true elementary low-energy excitations of the LM. The methods of Mattis and Lieb bear strong resemblance to the early work of Tomonaga [52] on the onedimensional electron gas. Extending the work of Tomonaga, as well as that of Mattis and Lieb, Luther and Peschel [53] computed the one- and two-particle correlation functions in equilibrium, thus showing that correlations exhibit (at zero temperature and long distances) a nonuniversal power-law behavior signaling the absence of long-range order. Later, Haldane [54-56] conjectured that these properties (i.e., collective elementary excitations exhausting the low-energy part of the spectrum as well as power-law correlations) are distinctive features of a large class of gapless interacting onedimensional systems that he termed (Tomonaga-) "Luttinger liquids." Using the modern language of critical phenomena, the LM can be understood as a fixed point of the renormalization group for a large class of gapless many-body systems in one dimension: the equilibrium properties at low temperatures of many $1 \mathrm{D}$ systems are universal in the sense that they can be accurately described by the LM. However, in this work, we shall be concerned with nonequilibrium properties of the LM and because the latter can involve highly excited states, we shall make no claim for universality. The precise conditions under which the results obtained here apply to real systems that are in the Tomonaga-Luttinger class should be investigated carefully in each particular instance (see discussion in Sec. IV).

The Hamiltonian of the LM can be written as follows:

$$
\begin{gathered}
H_{\mathrm{LM}}=H_{0}+H_{2}+H_{4}, \\
H_{0}=\sum_{p, \alpha=r, l} \hbar v_{F} p: \psi_{\alpha}^{\dagger}(p) \psi_{\alpha}(p):, \\
H_{2}=\frac{2 \pi \hbar}{L} \sum_{q} g_{2}(q): J_{r}(q) J_{l}(q): \\
H_{4}=\frac{\pi \hbar}{L} \sum_{q, \alpha=r, l} g_{4}(q): J_{\alpha}(q) J_{\alpha}(-q): .
\end{gathered}
$$

Here, $\psi_{\alpha}(p)$ and $\psi_{\alpha}^{\dagger}(p)$ are fermion creation and annihilation operators at some momentum $p$ and $J_{\alpha}(q)=\Sigma_{p}: \psi_{\alpha}^{\dagger}(p$ $+q) \psi_{\alpha}(p)$ :. The index $\alpha=r, l$ refers to the chirality of the fermion species, which can be either right $(r)$ or left $(l)$ moving; the symbol :...: stands for normal-ordering prescription for fermionic operators. This is needed to remove from the expectation values the infinite contributions arising from the fact that the ground state is a Dirac sea [55], namely, a state where all single-particle fermion levels with $p<0$ are occupied for both chiralities. This defines a stable ground state (at the noninteracting level), which in what follows will be denoted by $|0\rangle$.

\section{A. Bosonization solution of the LM}

In this section, we briefly review the solution of the LM. The Hamiltonian in Eqs. (1)-(4) can be written as a quadratic Hamiltonian in terms of a set of bosonic operators [51]. First, we note that the density operators $J_{\alpha}(q)=\Sigma_{p}: \psi_{\alpha}^{\dagger}(p$ $+q) \psi_{\alpha}(p)$ : obey the following commutation rules:

$$
\left[J_{\alpha}(q), J_{\beta}\left(q^{\prime}\right)\right]=\left(\frac{q L}{2 \pi}\right) \delta_{q+q^{\prime}, 0} \delta_{\alpha \beta},
$$

which can be transformed into the Heisenberg algebra of the familiar bosonic operators by introducing

$$
b(q)=-i\left(\frac{2 \pi}{|q| L}\right)^{1 / 2}\left[\vartheta(q) J_{r}(-q)-\vartheta(-q) J_{l}(q)\right],
$$

where $\vartheta(q)$ is the step function. Note that the $q=0$ components (known as "zero modes") require a separate treatment since $J_{\alpha}(0)=N_{\alpha}$ is the deviation, relative to the ground state, in the number of fermions of chirality $\alpha=r, l$. However, rather than working with $N_{r}$ and $N_{l}$, it is convenient to introduce

$$
N=N_{r}+N_{l} \quad J=N_{r}-N_{l},
$$

which, since $N_{r}$ and $N_{l}$ are integers, must obey the following selection rule $(-1)^{N}=(-1)^{J}$ when the Fermi fields obey antiperiodic boundary conditions $\psi_{\alpha}(x+L)=-\psi_{\alpha}(x) \quad(L$ is the length of the system). Therefore $\psi_{\alpha}(x)$ $=L^{-1 / 2} \Sigma_{p} e^{-a_{0}|p|} e^{i s_{\alpha} p x} \psi_{\alpha}(p)$, being $s_{r}=-s_{l}=+1$ and $p=2(n$ $\left.-\frac{1}{2}\right) \pi / L$, where $n$ is an integer and $a_{0} \rightarrow 0^{+}[55]$.

The Hamiltonian $H_{\mathrm{LM}}$ can be expressed in terms of the bosonic operators introduced in Eq. (6),

$$
\begin{aligned}
& H_{0}= \sum_{q \neq 0} \hbar v_{F}|q| b^{\dagger}(q) b(q)+\frac{\hbar \pi v_{F}}{2 L}\left(N^{2}+J^{2}\right), \\
& H_{2}= \frac{1}{2} \sum_{q \neq 0} g_{2}(q)|q|\left[b(q) b(-q)+b^{\dagger}(q) b^{\dagger}(-q)\right] \\
&+\frac{\hbar \pi g_{2}(0)}{2 L}\left(N^{2}-J^{2}\right), \\
& H_{4}=\sum_{q \neq 0} \hbar g_{4}(q)|q| b^{\dagger}(q) b(q)+\frac{\hbar \pi g_{4}(0)}{2 L}\left(N^{2}+J^{2}\right) .
\end{aligned}
$$

Ignoring the zero-mode part, the above Hamiltonian has the form of Eq. (A1), with the following identifications: 
$\omega_{0}(q)=v_{F}|q|, m(q, t)=g_{4}(q)|q|$, and $g(q, t)=g_{2}(q)|q|$, and it can be therefore be brought into diagonal form by means of the canonical transformation of Eq. (A3). Hence, the Hamiltonian takes the form of Eq. (A5) with $\omega(q)=v(q)|q|$, being $v(q)=\left\{\left[v_{F}+g_{4}(q)\right]^{2}-\left[g_{2}(q)\right]^{2}\right\}^{1 / 2}$ and $q \neq 0$. As to the zeromode contribution,

$$
H_{\mathrm{ZM}}=\frac{\hbar \pi v_{N}}{2 L} N^{2}+\frac{\hbar \pi v_{J}}{2 L} J^{2},
$$

where $v_{N}=v_{F}+g_{4}(0)+g_{2}(0)$ and $v_{J}=v_{F}+g_{4}(0)-g_{2}(0)$. This defines the equilibrium solution of the LM. In the following sections, we shall be concerned with the quench dynamics of this model.

\section{B. Suddenly turning on the interactions}

Although it is possible to solve the general quench problem between two interacting versions of the Luttinger model, we shall focus here on the cases where the interactions described by $H_{2}$ and $H_{4}$ [cf. Eqs. (3) and (4)] are suddenly switched on (this section) and switched off (next section). Thus, in this section, we shall assume that we have made the replacements $g_{2,4}(q) \rightarrow g_{2,4}(q, t)=g_{2,4}(q) \theta(t)$ in Eqs. (3) and (4). The Hamiltonian at times $t>0$ is therefore the interacting LM. In other words, using the notation introduced in Appendix A, $H_{f}=H_{0}+H_{2}+H_{4}=H_{\mathrm{LM}}$, whereas the initial Hamiltonian (for $t \leq 0$ ) is $H_{i}=H_{0}$. However, we note that, since both zero modes, $J$ and $N$, are conserved by $H_{i}=H_{0}$ and $H_{f}=H_{\mathrm{LM}}$, their dynamics factors out and we shall assume henceforth that we work within the sector of the Hilbert space where $J=N=0$ (this sector contains the noninteracting ground state, $|0\rangle)$. Therefore, from now on, we shall omit $H_{\mathrm{ZM}}$ in all discussions.

As to the initial state, we shall consider that, within the spirit of the sudden approximation, at $t=0$, the system is prepared in a Boltzmann ensemble at a temperature $T$ described by

$$
\rho_{0} \equiv \rho(t=0)=Z_{0}^{-1} e^{-H_{i} / T},
$$

where $Z_{0}=\operatorname{Tr} e^{-H_{i} / T}$. We shall further assume that the contact with the reservoir is removed at $t=0$ and that, after the quench, the system undergoes a unitary as it is in isolation from the rest of the universe.

Equation (A7) describes the solution to the interaction quench in terms of the modes that annihilate the initial ground state $|0\rangle$; the solution itself is not particularly illuminating. To gain some insight into the properties of the system following the quench, let us compute a few observables. Among them, we first turn our attention to the instantaneous momentum distribution, which is the Fourier transform of the one-particle density matrix

$$
C_{\psi_{r}}(x, t)=\left\langle e^{i H_{f} t / \hbar} \psi_{r}^{\dagger}(x) \psi_{r}(0) e^{-i H_{f} t / \hbar}\right\rangle_{0},
$$

where $\langle\cdots\rangle_{0}$ means that the expectation value is taken over the ensemble described by $\rho_{0}$ [cf. Eq. (12)]. The time dependence of the operators is dictated by $H_{f}$, as described in Appendix A. Notice that, since in general $\left[H_{f}, \rho_{0}\right] \neq 0$, time translation invariance is broken and the above correlation function is explicitly time dependent.
The time evolution of $\psi_{\alpha}(x)$ can be obtained using the bosonization formula for the field operator [53,55,57]

$$
\psi_{\alpha}(x)=\frac{\eta_{\alpha}}{\sqrt{2 \pi a}} e^{i s_{\alpha} \phi_{\alpha}(x)},
$$

being $\eta_{r}, \eta_{l}$ two Majorana operators (also known as Klein factors) obeying $\left\{\eta_{\alpha}, \eta_{\beta}\right\}=2 \delta_{\alpha \beta}$, which ensures the anticommutation of the left- and right-moving Fermi fields (recall that $s_{\alpha}=+1$ for $\alpha=r$ and $s_{\alpha}=-1$ for $\alpha=l$ ). The bosonic fields

$$
\phi_{\alpha}(x)=s_{\alpha} \varphi_{0 \alpha}+\frac{2 \pi x}{L} N_{\alpha}+\Phi_{\alpha}^{\dagger}(x)+\Phi_{\alpha}(x)
$$

where $\left[N_{\alpha}, \varphi_{0 \beta}\right]=i \delta_{\alpha, \beta}$ and, in terms of Fourier modes,

$$
\Phi_{\alpha}(x)=\lim _{a_{0} \rightarrow 0^{+}} \sum_{q>0}\left(\frac{2 \pi}{q L}\right)^{1 / 2} e^{-q a_{0} / 2} e^{i s_{\alpha} q x} b\left(s_{\alpha} q\right) .
$$

The details of the calculations of $C_{\psi_{r}}(x, t)$ have been relegated to the Appendix B. In this section, we will mainly describe the results. However, a number of remarks about how the calculations were performed are in order before proceeding any further. We first note that interactions in the LM are assumed to be long ranged $[50,51,55]$. This can be made explicit in the interaction couplings by writing $g_{2,4}(q)$ $=g_{2,4}\left(q R_{0}\right)$, where the length scale $R_{0} \ll L$ is the interaction range. Thus, just like system size $L$ plays the role of a cutoff for "infrared" (that is, long wavelength) divergences, the interaction range $R_{0}$ plays the role of an "ultraviolet" cutoff that regulates the short-distance divergences of the model. The results given below were derived assuming a particular form of the interaction (or regularization scheme) where the Bogoliubov parameter [cf. Eq. (A4)] is chosen such that $\sinh 2 \beta(q)=\gamma e^{-\left|q R_{0}\right| / 2}$. Furthermore, we replaced $v(q)$ by $v$ $=v(0)$. Indeed, these approximations are fairly similar to the ones used to compute the time-dependent correlation functions in equilibrium [53], given that the expressions that we obtain for the out-equilibrium correlators are fairly similar to those of the equilibrium correlations [53]. This regularization scheme greatly simplifies the calculations while not altering in a significant way the asymptotic behavior of the correlators for distances much larger than $R_{0}{ }^{3}$

Returning to the one-particle density matrix [cf. Eq. (13)], we note that it can be written as the product of two factors:

$$
C_{\psi_{r}}(x, t)=C_{\psi_{r}}^{(0)}(x) h_{r}(x, t)
$$

where $C_{\psi_{r}}^{(0)}(x)$ is the noninteracting one-particle density matrix and thus $h_{r}(x, t)$ accounts for deviations due to the interactions. Hence, this factorization allows us to obtain the instantaneous momentum distribution function as the convolution

\footnotetext{
${ }^{3}$ An exception are pathological cases, such as the Coulomb interaction, where both $v(q) /|q|$ and $\sinh 2 \beta(q)$ are singular for $q=0$.
} 


$$
f(p, t)=\int_{-\infty}^{\infty} \frac{d k}{2 \pi} f^{(0)}(p-k) h_{r}(k, t)
$$

where $f^{(0)}(p)=\left(e^{\hbar v_{F} p / T}+1\right)^{-1}$ is the Fermi-Dirac distribution and

$$
h_{r}(k, t)=\int_{-\infty}^{+\infty} d x e^{i p x} h_{r}(x, t)
$$

Before presenting the results for the expression of the one-particle density matrix as well as the momentum distribution at finite temperatures, it is worth considering the much simpler-looking zero-temperature expression. We first discuss finite-size effects. For a system of size $L$, we obtain [9]

$$
\begin{aligned}
C_{\psi_{r}}(x \mid L)= & C_{\psi_{r}}^{(0)}(x \mid L) \\
& \times\left|\frac{R_{0}}{d(x \mid L)}\right| \gamma^{2}\left|\frac{d(x-2 v t \mid L) d(x+2 v t \mid L)}{d(2 v t \mid L) d(-2 v t \mid L)}\right| \gamma^{2} / 2,
\end{aligned}
$$

where $d(z \mid L)=L|\sin (\pi z / L)|$ is the cord function and $C_{\psi_{r}}^{(0)}(x \mid L)=i\left\{2 L \sin \left[\pi\left(x+i a_{0}\right) / L\right]\right\}^{-1}\left(a_{0} \rightarrow 0^{+}\right)$the noninteracting one-particle density matrix. Notice that this result is valid only asymptotically, that is, for $d(x \mid L), d(x \pm 2 v t)$ $\gg R_{0}$. Thus, we see that $C_{\psi_{r}}(x, t \mid L)$ is a periodic function of time with period equal to $\tau_{0}=L / 2 v$. This is in agreement with the general expectation that correlations in finite-size systems exhibit time recurrences because the energy spectrum is discrete. Although the recurrence time generally depends on the details of the energy spectrum, in the LM, the spectrum is linear $\omega(q) \simeq v|q|$ and therefore the energy spacing between (nondegenerate) many-body states is $\Delta_{0}$ $\simeq 2 \pi \hbar v / L$. Hence, the recurrence time $\tau_{0} \sim 2 \pi / \Delta_{0}$ follows (the extra factor of $\frac{1}{2}$ is explained by the so-called light-cone effect, see further below). The recurrent behavior exhibited by the one-particle density matrix (13) implies that, after the quench, the system does not reach a time-independent stationary state as time grows. A similar conclusion is reached by analyzing other correlations such as, e.g., the finite-size version of the density-correlation function [9],

$$
\begin{aligned}
C_{J_{r}}(x, t \mid L)= & \left\langle e^{i H_{f} t / \hbar} J_{r}(x) J_{r}(0) e^{-i H_{f} t / \hbar}\right\rangle_{0} \\
= & -\frac{\left(1+\gamma^{2}\right) / 4 \pi^{2}}{[d(x \mid L)]^{2}}+\frac{\gamma^{2} / 8 \pi^{2}}{[d(x-2 v t \mid L)]^{2}} \\
& +\frac{\gamma^{2} / 8 \pi^{2}}{[d(x+2 v t \mid L)]^{2}},
\end{aligned}
$$

where

$$
J_{r}(x)=\sum_{q} \frac{e^{i q x}}{L} J_{r}(q)=: \psi_{r}^{\dagger}(x) \psi_{r}(x)=: \frac{1}{2 \pi} \partial_{x} \phi_{r}(x)
$$

is the density (also referred to as "current") operator in real space.
In the thermodynamic limit, $L \rightarrow \infty$, the recurrence time $\tau_{0}=L / 2 v \rightarrow+\infty$ and the system does indeed reach a timeindependent steady state. In this limit, $d(x \mid L) \rightarrow|x|$ and the single-particle density matrix becomes [9]

$$
C_{\psi_{r}}(x, t>0)=G_{r}^{(0)}(x)\left|\frac{R_{0}}{x}\right| \gamma^{2}\left|\frac{x^{2}-(2 v t)^{2}}{(2 v t)^{2}}\right|^{\gamma^{2} / 2} .
$$

Hence,

$$
h_{r}(x, t)=\left|\frac{R_{0}}{x}\right| \gamma^{\gamma^{2}}\left|\frac{x^{2}-(2 v t)^{2}}{(2 v t)^{2}}\right| \gamma^{2} / 2 .
$$

In order to understand the evolution of the momentum distribution, without actually having to compute it, it is useful to consider the various limits of the above expression, Eq. (23). First of all, for short times such that $2 v t \ll|x|$, the function $h_{r}(x, t)$ is asymptotically just a time-dependent factor [9]

$$
h_{r}(x, t) \simeq Z(t)=\left(\frac{R_{0}}{2 v t}\right)^{\gamma^{2}},
$$

which can be interpreted as a time-dependent "Landau quasiparticle" weight in an effective (time-dependent) Fermiliquid description of the system. In other words, as time evolves after the quench, we could imagine that the quasiparticle weight at the Fermi level is reduced from its initial value, $Z(t=0)=1$, to $Z(t>0)<1$. At zero temperature, this time-dependent renormalization of the quasiparticle weight reflects itself in a reduction of the discontinuity of the momentum distribution $f(p, t)$ at the Fermi level (which is located at $p=0$ in our convention). Therefore, at any finite time, the system behaves as if it was a Fermi liquid and therefore it keeps memory of the initial state, that is, a noninteracting Fermi gas.

Yet, for $t \rightarrow+\infty, h_{r}(x, t)$ becomes a power law [9]

$$
\lim _{t \rightarrow \infty} h_{r}(x, t)=\left|\frac{R_{0}}{x}\right|^{\gamma^{2}},
$$

and also does the momentum distribution. The behavior of the momentum distribution at different times is depicted in Fig. 1. Interestingly, this time-dependent reduction of the quasiparticle weight after being quenched into the interacting state has been also found in Ref. [20] when studying an interaction quench in the Hubbard model in the limit of infinite dimensions. In this case, however, the discontinuity remains finite even for $t \rightarrow+\infty$, which is different from the behavior of the LM, which is known to be a non-Fermi liquid at equilibrium. Some of these non-Fermi-liquid features also persist in the quench dynamics.

The result of Eq. (26) is similar to the zero-temperature result in equilibrium. However, the exponent of $C_{\psi_{r}}(x, t$ $\rightarrow \infty$ ) is equal to $1+\gamma^{2}$ and, even for an infinitesimal interaction (i.e., $\gamma \ll 1$ ), it is always larger than the one that governs the ground state (i.e., equilibrium) correlations $[53,55]$ : $\gamma^{2}=\sinh ^{2} 2 \beta(0)>\gamma_{\text {eq }}^{2}=2 \sinh ^{2} \beta(0)$. The reason for the larger exponent can be qualitatively understood from the following facts: (i) Because of the variational theorem, the initial state (i.e., the ground state of the noninteracting Hamiltonian $\left.H_{i}=H_{0}\right)$ is a complicated excited state of the final 


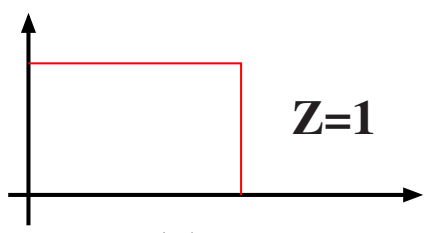

(a)

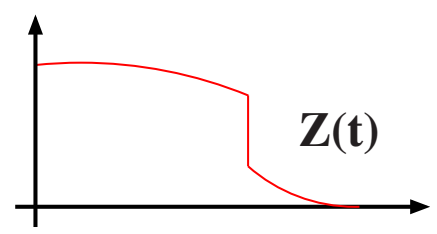

(b)

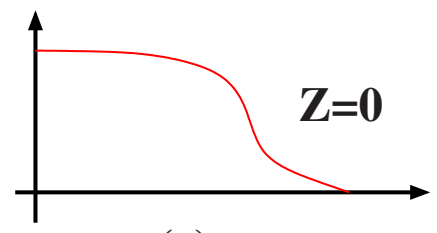

(c)

FIG. 1. (Color online) Schematic time evolution of the momentum distribution $f(p, t)$ at zero temperature. (a) At $t=0$, the momentum distribution is that of noninteracting fermions, with a discontinuity at the Fermi level $(p=0) Z=1$. (b) At $t>0$, the discontinuity is reduced in a power-law fashion $Z(t) \sim t^{-\gamma^{2}}$. (c) For $t \rightarrow+\infty$, the discontinuity disappears and the momentum distribution exhibits a power-law singularity close to the Fermi level $p=0, f(p, t \rightarrow+\infty)$ $=\frac{1}{2}-$ const $|p|^{\gamma^{2}}$. However, the exponent characterizing the singularity is not the equilibrium exponent.

Hamiltonian $H_{f}=H_{\mathrm{LM}}$. (ii) Both $H_{i}$ and $H_{f}$ are critical (i.e., scale free, apart from the cutoff $R_{0}$ ), thus, the system is likely to remain critical. To end the present discussion, we note that, in the literature on Tomonaga-Luttinger liquids, it is customary to introduce the dimensionless parameter $K$ $=e^{-2 \beta(0)}$ in terms of which $\gamma^{2}=\left(K-K^{-1}\right)^{2} / 4$, which needs to be compared to the equilibrium exponent resulting from $\gamma_{\text {eq }}=\left(K+K^{-1}-2\right) / 2$. We shall use the parameter $K$ in other expressions below.

It is worth emphasizing that the particular evolution of the asymptotic correlations from Fermi-liquid-like at short times to non-Fermi-liquid-like at infinite time exhibited by the oneparticle density matrix is also found in other correlation functions. However, the idea that the system "looks like" an interacting Fermi liquid at any finite $t$ should not be taken too far. In this regard, we should note that for $|x| \gg 2 v t$, the prefactor of the term $\propto(2 \pi x)^{-2}$ of the density-correlation function [cf. Eq. (21)], which in equilibrium is proportional to the system compressibility [57], remains equal to (minus) unity, which is the value that corresponds to a noninteracting Fermi gas (in an interacting Fermi liquid, it would deviate from one). For $t \rightarrow+\infty$, the same prefactor becomes $\left(1+\gamma^{2}\right)>1$, which does not relate easily to a non-Fermi-liquid-like behavior. However, other correlation functions exhibit a similar behavior to the one-particle density matrix. For instance, let us consider the following correlators:

$$
C_{\phi}^{m}(x, t)=\left\langle e^{2 i m \phi(x, t)} e^{-2 i m \phi(0, t)}\right\rangle,
$$

$$
C_{\theta}^{n}(x, t)=\left\langle e^{i n \theta(x, t)} e^{-i n \theta(0, t)}\right\rangle,
$$

where $\phi(x)=\frac{1}{2}\left[\phi_{r}(x)+\phi_{l}(x)\right]$ and $\theta(x)=\frac{1}{2}\left[\phi_{r}(x)-\phi_{l}(x)\right]$ and the spatial derivatives of $\phi$ and $\theta$ are related to the (total) density and current-density fluctuations, respectively. ${ }^{4}$ Using exactly the same methods as above, we find (for $L \rightarrow \infty$ )

$$
\begin{aligned}
& \frac{C_{\phi}^{m}(x, t)}{C_{\phi}^{(0, m)}(x)}=\left|\left(\frac{R_{0}}{2 v t}\right)^{2} \frac{x^{2}-(2 v t)^{2}}{x^{2}}\right| m^{2}\left(K^{2}-1\right) / 2, \\
& \frac{C_{\phi}^{n}(x, t)}{C_{\phi}^{(0, n)}(x)}=\left|\left(\frac{R_{0}}{2 v t}\right)^{2} \frac{x^{2}-(2 v t)^{2}}{x^{2}}\right|^{n^{2}\left(K^{-2}-1\right) / 8},
\end{aligned}
$$

where $C^{(0, m)}(x)=A_{m}^{\phi}\left|R_{0} / x\right|^{2 m^{2}}$ and $C^{(0, n)}(x)=A_{n}^{\theta}\left|R_{0} / x\right|^{n^{2} / 2}$ are the noninteracting correlation functions (where $A_{m}^{\phi}$ and $A_{n}^{\theta}$ are nonuniversal prefactors). We note that the usual duality relation where $\phi \rightarrow \theta$ and $K \rightarrow K^{-1}$, which one encounters when studying equilibrium correlation functions [57], still holds for these nonequilibrium correlators. Let us next analyze their asymptotic properties. We consider only $C_{\phi}^{m}(x, t)$, as identical conclusions also apply to $C_{\theta}^{n}(x, t)$ by virtue of the duality relation. For $|x| \gg 2 v t$, we have

$$
C_{\phi}^{m}(x, t)=C^{(0, m)}(x)\left(\frac{R_{0}}{2 v t}\right)^{m^{2}\left(K^{2}-1\right)} .
$$

Thus, up to the time-dependent prefactor, correlations take the form of a noninteracting system of Fermions, $C^{(0, m)}(x)$. However, in the opposite limit $(|x| \ll 2 v t)$, this correlator exhibits a nontrivial power law

$$
C_{\theta}^{m}(x, t) \simeq\left|\frac{R_{0}}{x}\right|^{m^{2}\left(K^{2}+1\right)} .
$$

Notice that this expression also describes the infinite-time behavior, which is controlled by an exponent equal to $m^{2}\left(K^{2}+1\right)$, being again different from the exponent exhibited by the same correlator in equilibrium, which equals $2 m^{2}[\cosh 2 \beta(0)-\sinh 2 \beta(0)]=2 m^{2} K$.

In order to understand why the behavior found for $t \rightarrow \infty$ in Eqs. (26) and (32) also holds for $|x| \ll 2 v t$, let us consider the initial state at zero temperature, $\rho_{0}=|0\rangle\langle 0|[12,15]$. As mentioned above, this is a rather complicated excited state of the Hamiltonian that performs the time evolution, $H_{f}=H_{\mathrm{LM}}$.

\footnotetext{
${ }^{4}$ In a Luttinger liquid, the $C_{\phi}^{m}$ correlator describes the fluctuations of wave number close to $2 m k_{F}$ (where $k_{F}$ is the Fermi momentum) of the density-correlation function [55,57]. In the presence of a periodic potential of periodicity equal to $2 m k_{F}$, the system may become an insulator [57]. The power-law behavior exhibited at zero temperature (and in the thermodynamic limit) is a consequence of the gapless spectrum and the absence of long-range order in the density. In the insulating (i.e., gapped) phase, this correlation function decays to a nonzero constant at long distances, which is a consequence of the existence of long-ranged order in the density at wave number $2 m k_{F}$. Similarly, in equilibrium, $C_{\theta}^{n}$ measures the phase fluctuations and exhibits a power law, reflecting the absence of long-range order in the phase. However, in the Luttinger model of interest for us here, terms with $m>0$ are absent from the density operator, which is given by $\rho(x)=J_{r}(x)+J_{l}(x)[55]$.
} 
This means that, initially, there are a large number of excitations of $H_{f}$, namely, phonons with dispersion $\omega(q)$ $=v(q)|q|$. The distribution of the phonons $\left\langle b^{\dagger}(q) b(q)\right\rangle_{0}$ $=\sinh ^{2} \beta(q)$ is time independent and peaked at $q=0$. Thus, within the approximation where $v(q) \simeq v(0)=v$, the excitations propagate between two given points with velocity $v$. Thus, if we consider the correlations at two points $A$ and $B$ separated by a distance $|x|$, the nature of the correlation at a given time $t$ depends on whether the excitations found initially at, say, point $A$, have been able to reach point $B$ or not. This is not the case if $|x|>2 v t$ and thus correlations retain essentially the properties they had in the initial state. Thus, up to a time-dependent prefactor, $C_{\psi_{r}}(x, t) \propto C_{\psi_{r}}^{(0)}(x)$. However, if the two points have been able to "talk to each other" through the excitations present in the initial state, then correlations will be qualitatively different. This happens for a time $t=t_{0}$ when the excitations propagating from $A$ meet the excitations propagating from $B$, that is, for $x-v t_{0}=v t_{0}$ or $t_{0}$ $=x / 2 v$ (we assume $x>0$ without loss of generality). Thus, for given separation $x$ and time $t$, there is a length scale $2 v t$, which marks the transition between two different regimes in the correlations. In the instantaneous momentum distribution, this reflects itself in a crossover as a function of time from a momentum distribution $n(p)$ exhibiting a discontinuous Fermi-liquid-like behavior, which is valid, i.e., for $|p|$ $\ll(2 v t)^{-1}$ to a power-law behavior of the form $\sim\left|p R_{0}\right| \gamma^{2} \operatorname{sgn}(p)$, which applies for $|p| \gg(2 v t)^{-1}$ but $|p|$ $\ll R_{0}^{-1}$ (for $|p| \gg R_{0}^{-1}$, we recover the free-particle behavior corresponding to the Fermi-Dirac distribution function at $T$ $=0$ ). In the $t \rightarrow \infty$ limit, by using the regularization scheme described above, the asymptotic momentum distribution at zero temperature can be obtained with the help of tables [58]. The resulting formula behaves as the noninteracting FermiDirac distribution for $|p| \gg R_{0}^{-1}$, whereas for $|p| \gg R_{0}^{-1}$, it describes a non-Fermi-liquid-like steady state

$$
\begin{aligned}
f(p, t \rightarrow & +\infty)=\frac{1}{2}-\frac{p R_{0}}{2}\left[K_{\gamma^{2}-1 / 2}\left(\left|p R_{0}\right|\right) \mathcal{L}_{\gamma^{2}-3 / 2}\left(\left|p R_{0}\right|\right)\right. \\
& \left.+K_{\gamma^{2}-3 / 2}\left(\left|p R_{0}\right|\right) \mathcal{L}_{\gamma^{2}-1 / 2}\left(\left|p R_{0}\right|\right)\right]
\end{aligned}
$$

where $K_{\nu}(z)$ and $\mathcal{L}_{\nu}(z)$ are the modified Bessel and Struve functions [58], respectively. This expression yields a power law for $\left|p R_{0}\right| \ll 1$, where $n(p, t \rightarrow+\infty) \simeq \frac{1}{2}$ $-\operatorname{const}\left(\left|p R_{0}\right|\right) \gamma^{2} \operatorname{sgn}(p)$. Note that the momentum distribution $n(p=0, t)=\frac{1}{2}$, which is given by the invariance of the LM under particle-hole symmetry $\psi_{\alpha}(p) \rightarrow \psi_{\alpha}^{\dagger}(-p)$.

Let us finally present the generalization of the above results for the one-particle density matrix to finite temperatures, $T>0$. For $T \ll \hbar v_{F} R_{0}$ (but $T \gg \Delta_{0}=2 \pi \hbar v / L$, so that we can neglect finite-size effects and effectively take the thermodynamic limit) $C_{\psi_{r}}(x, t)$ takes the following asymptotic form:

$$
\begin{aligned}
& C_{\psi_{r}}(x, t>0 \mid T)=C_{\psi_{r}}^{(0)}(x \mid T) \\
& \times\left|\frac{\pi R_{0} / \lambda}{d h(x \mid T)}\right| \gamma^{2}\left|\frac{d h(x-2 v t \mid T) d h(x+2 v t \mid T)}{d h(2 v t \mid T) d h(-2 v t \mid T)}\right|^{\gamma^{2} / 2},
\end{aligned}
$$

where $C_{\psi_{r}}^{(0)}(x \mid T)$ and $d h(x \mid T)$ can be obtained from $C_{\psi_{r}}^{(0)}(x \mid L)$ and $d(x \mid L)$ by replacing $L \sin (\pi x / L) / \pi$ by $\lambda \sinh (\pi x / \lambda)$, where $\lambda=\hbar v_{F} / T$ is the thermal correlation length. At long times, $h_{r}(x, t \mid T)$ reduces to

$$
h_{r}(x)=\left|\frac{\pi R_{0} / \lambda}{\sinh (\pi x / \lambda)}\right| \gamma^{2} .
$$

Therefore, we again find that $C_{\psi_{r}}(x, t \rightarrow \infty \mid T)$ has a form similar to the equilibrium correlation function at finite temperature with a different exponent controlling the asymptotic exponential decay of correlations. Notice that the exponential decay in the correlations for $t>0$ is a direct consequence of the fact that the initial state has a characteristic correlation length, the thermal correlation length $\lambda=\hbar v_{F} / T .^{5}$ The exponential decay of correlations at finite $T$ implies that the steady state will be reached exponentially rapidly in a time of the order of $\hbar / T$. It is also worth noting, however, that the above expression depends parametrically on the thermal correlation length $\lambda$ and the Fermi velocity, $v_{F}$, which enters in the expression for $\lambda=\hbar v_{F} / T$, instead of the (renormalized) phonon velocity which enters in the thermal length $\lambda_{\text {eq }}$ $=\hbar v / T$, characterizing equilibrium correlations. Thus, since the velocity appears only through the definition of the thermal correlation length $\lambda$ or, in other words, in combination with the temperature, the change from $v$ to $v_{F}$ can be also understood as an change in the temperature scale. Furthermore, in a system with Galilean symmetry [57], we have that $v K=v_{F}$ and thus the parameter that controls the temperature scale now is the Luttinger parameter $K$, so that the asymptotic correlations at $t \rightarrow \infty$ can be regarded as the equilibrium correlations with a different exponent and an effective temperature, $T_{\text {eff }}=T / K$. Thus, for repulsive interactions (i.e., $K<1$ ) we could say that, besides modifying the exponent, the interaction quench increases the effective temperature, whereas for attractive interactions (i.e., $K>1$ ), the effective temperature is reduced after the quench. This effect has an impact on the momentum distribution at finite temperatures because it compensates the effect of the larger nonequilibrium exponent on the momentum distribution at finite $T$. To show this, we need to obtain the Fourier transform of $h_{r}(x)$. This can be done by relating it to an integral representation of the associated Legendre function $P_{\mu}^{\nu}(z)$ [58] and thus the Fourier transform of $h_{r}(x)$ can be written as

$$
\begin{aligned}
h_{r}(p)= & \frac{\lambda}{\sqrt{\pi}}\left(\frac{\pi R_{0}}{\lambda}\right)^{\left(\gamma^{2}+1\right) / 2} \frac{\left|\Gamma\left(\frac{\gamma^{2}}{2}+\frac{i \lambda p}{2 \pi}\right)\right|^{2}}{\Gamma\left(\frac{\gamma^{2}}{2}\right)} \\
& \times P_{i \lambda p / 2 \pi-1 / 2}^{-\gamma^{2} / 2+1 / 2}\left[-\cos \left(\frac{2 \pi R_{0}}{\lambda}\right)\right] .
\end{aligned}
$$

Hence, the momentum distribution can be obtained by numerically evaluating the convolution with the Fermi-Dirac

\footnotetext{
${ }^{5} \mathrm{~A}$ similar situation is found when analyzing quenches at $T=0$ from a noncritical (that is, gapped) into a critical (that is, gapless) state. In that case, the role of $\lambda$ will be played by the correlation length of the system that is determined by the (inverse of the) energy gap in the initial state $[12,48]$.
} 


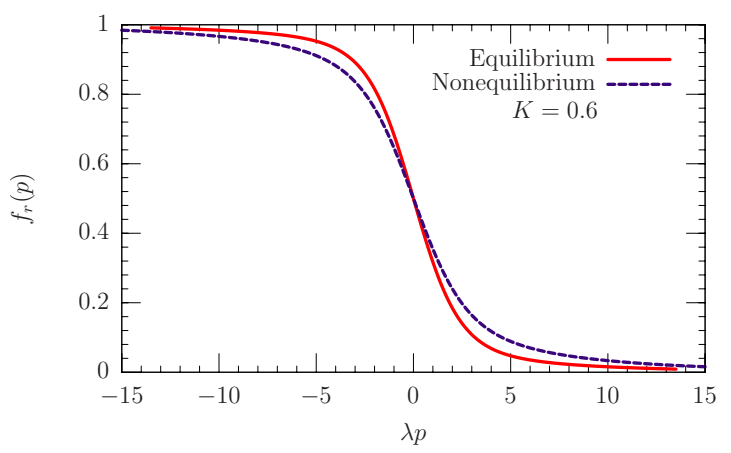

FIG. 2. (Color online) Infinite time limit of the momentum distribution vs $\lambda p\left(\lambda=\hbar v_{F} / T\right.$ is the thermal correlation length in the initial state) for a noninteracting Luttinger model at finite temperature $T$ that is quenched into an interacting state with repulsive interactions (corresponding to a Luttinger parameter $K=0.6$ ).

distribution function [cf. Eq. (18)] of the above expression, Eq. (36). In Figs. 2 and 3, the momentum distribution of the interacting system for $t \gg \hbar / T$ is displayed for a noninteracting LM that undergoes an interaction quench with repulsive (corresponding to $K=0.6$ ) and attractive (corresponding to $K=1.7)$ interactions, respectively.

\section{Suddenly turning off the interactions}

Next, we briefly consider the opposite situation to the one analyzed above, namely, the case where interaction between the fermions in the initial state suddenly disappears. The fact that the initial state is a highly complicated state of the Hamiltonian that performs the time evolution [in this case $H_{f}=H_{0}$, cf. Eq. (1)], implies that we cannot expect that a Fermi liquid will emerge asymptotically at long times after the quench. Indeed, at zero temperature, a thermodynamically large system approaches a steady state exhibiting equaltime correlations that decay algebraically in space. However, the exponents differ again from the (noninteracting) equilibrium ones. This can be illustrated by, e.g., computing the following correlation functions:

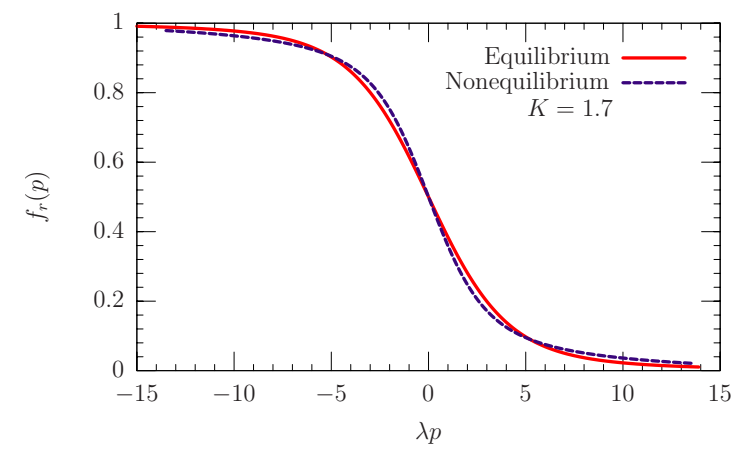

FIG. 3. (Color online) Infinite time limit of the momentum distribution vs $\lambda p\left(\lambda=\hbar v_{F} / T\right.$ is the thermal correlation length in the initial state) for a noninteracting Luttinger model at finite temperature $T$ that is quenched into an interacting state with attractive interactions (corresponding to a Luttinger parameter $K=1.7$ ).

$$
\begin{aligned}
C_{\phi}^{m}(x, t) & =\left\langle e^{2 i m \theta(x, t)} e^{-2 i m \theta(0, t)}\right\rangle \\
& =I_{\phi}^{m}(x)\left(\frac{R_{0}}{2 v_{F} t}\right)^{m^{2}\left(K^{-1}-K\right)}\left|\frac{x^{2}-\left(2 v_{F} t\right)^{2}}{x^{2}}\right| m^{2}\left(K^{-1}-K\right) / 2 \\
C_{\theta}^{n}(x, t) & =\left\langle e^{i n \phi(x, t)} e^{-i n \phi(0, t)}\right\rangle \\
& =I_{\theta}^{n}(x)\left(\frac{R_{0}}{2 v_{F} t}\right)^{n^{2}\left(K-K^{-1}\right) / 2}\left|\frac{x^{2}-\left(2 v_{F} t\right)^{2}}{x^{2}}\right| n^{2}\left(K-K^{-1}\right) / 4
\end{aligned}
$$

where

$$
\begin{aligned}
& I_{\phi}^{m}(x)=\left|\frac{R_{0}}{x}\right|^{2 m^{2} K}, \\
& I_{\theta}^{n}(x)=\left|\frac{R_{0}}{x}\right|^{n^{2} / 2 K}
\end{aligned}
$$

are the correlation functions in the initial (interacting) ground state $\left(A_{\theta / \phi}\right.$ are nonuniversal prefactors). We note again that the duality $\theta \rightarrow \phi$ and $K \rightarrow K^{-1}$ also holds in this case. The correlations in the stationary state that is asymptotically approached at long times read

$$
\begin{aligned}
& \lim _{t \rightarrow+\infty} C_{\theta}^{m}(x, t)=A_{\theta}\left|\frac{R_{0}}{x}\right|^{m^{2}\left(K^{-1}+K\right)}, \\
& \lim _{t \rightarrow+\infty} C_{\phi}^{n}(x, t)=A_{\phi}\left|\frac{R_{0}}{x}\right|^{n^{2}\left(K^{-1}+K\right) / 2} .
\end{aligned}
$$

However, at short times, $t \ll|x| / 2 v_{F}$, correlations look like those of in the initial state up to a time-dependent prefactor

$$
\begin{aligned}
& C_{\theta}^{m}\left(x, t \ll|x| / 2 v_{F}\right)=\left(\frac{R_{0}}{2 v_{F} t}\right)^{m^{2}\left(K^{-1}-K\right)} \quad I_{\theta}^{m}(x), \\
& C_{\phi}^{n}\left(x, t \ll|x| / 2 v_{F}\right)=\left(\frac{R_{0}}{2 v_{F} t}\right)^{n^{2}\left(K-K^{-1}\right) / 2} \\
& I_{\phi}^{n}(x) .
\end{aligned}
$$

In this case, the time-dependent prefactor has also a powerlaw form.

\section{LONG-TIME DYNAMICS AND THE GENERALIZED GIBBS ENSEMBLE}

Recently, Rigol and co-workers [10] observed that, at least for observables such as the momentum distribution or the ground-state density, their long-time behavior following a quantum quench in an integrable system can described by adopting the maximum entropy (also called "subjective") approach to statistical mechanics pioneered by Jaynes $[45,46,59]$. Within this approach, the equilibrium state of a system is described by a density matrix that extremizes the von Neumann entropy, $S=-\operatorname{Tr} \rho \ln \rho$, subject to the constraints provided by a certain set of integrals of motion of the 
system. In the case of an integrable system, if $\left\{I_{m}\right\}$ is a set of certain (but not all of the possible) independent integrals of motion of the system, this procedure leads to a "generalized" Gibbs ensemble, described by the following density matrix:

$$
\rho_{\mathrm{gG}}=\frac{1}{Z_{\mathrm{gG}}} \exp \left(-\sum_{m} \lambda_{m} I_{m}\right),
$$

where $Z_{\mathrm{gG}}=\operatorname{Tr} \exp \left(-\Sigma_{m} \lambda_{m} I_{m}\right)$. The values of the Lagrange multipliers $\lambda_{m}$ must be determined from the condition that

$$
\left\langle I_{m}\right\rangle_{\mathrm{gG}}=\operatorname{Tr}\left[\rho_{0} I_{m}\right]=\left\langle I_{m}\right\rangle,
$$

where $\rho_{0}$ describes the initial state of the system and $\langle\cdots\rangle_{\mathrm{gG}}$ stands for the average taken over the generalized Gibbs ensemble, Eq. (45). Although $\rho_{i}=|\Phi(t=0)\rangle\langle\Phi(t=0)|$ in the case of a pure state, as was first used in Ref. [10], nothing prevents us from taking $\rho_{0}$ to be an arbitrary mixed state and in particular a thermal state characterized by an absolute temperature $T$. In such a case, the Lagrange multipliers will depend on $T$ or any other parameter that defines the initial state.

Rigol and co-workers tested numerically the above conjecture by studying the quench dynamics of a 1D lattice gas of hard-core bosons (see Refs. $[10,13]$ for more details). The question that naturally arises then is whether correlations of the LM also relax in agreement with the above conjecture. In other words, does the average $\langle O\rangle(t)$ at long times relax to the value $\langle O\rangle_{\mathrm{gG}}=\operatorname{Tr} \rho_{\mathrm{gG}} O$ for any of the correlation functions considered previously? We shall first discuss an observable for which the generalized Gibbs ensemble fails to reproduce their expectation values. Moreover, by considering the correlation function of the current operators [i.e., $O=J_{r}(x)$, cf. Eq. (22)], we will illustrate why it works in the LM. Calculations of other observables can be found in Appendixes A and $\mathrm{C}$.

Let us define the generalized Gibbs ensemble for the LM. Since the final Hamiltonian (in the $N=0$ and $J=0$ sectors) is diagonal in the $b$ boson operator basis, i.e., $H_{\mathrm{LM}}$ $=\Sigma_{q \neq 0} \hbar v(q)|q| b^{\dagger}(q) b(q)$, a natural choice for the set of integrals of motion is $I_{m} \rightarrow I(q)=n(q)=b^{\dagger}(q) b(q)$ for all $q \neq 0$ (a more complete version of the ensemble should also include $N$ and $J$, but this will not be necessary as we work in the thermodynamic limit). Thus, for the quench from the noninteracting to the interacting state (cf. Sec. II B), where the initial state is $|\Phi(t=0)\rangle=|0\rangle$, the Lagrange multipliers are determined by Eq. (46), which yields

$$
\langle I(q)\rangle_{\mathrm{gG}}=\langle n(q)\rangle_{\mathrm{gG}}=\sinh ^{2} \beta(q)=\frac{1}{e^{\lambda(q)}-1} .
$$

Indeed, this result can be quickly established by realizing that $\rho_{\mathrm{gG}}$ has the same form as the density matrix of a peculiar canonical ensemble where the temperature on each eigenmode of the final Hamiltonian depends on the wave-vector $q$, that is, $T(q)=\hbar v(q)|q| / \lambda(q)$. Alternatively, one can also regard it as an ensemble where the effective Hamiltonian that defines the Boltzmann weight is given by $H_{\text {eff }} / T_{\text {eff }}$ $=\sum_{q \neq 0} \lambda(q) n(q)$. However, it is worth noting that $\rho_{\mathrm{gG}}$ is diagonal in $n(q)$ and therefore it does not capture the correlations existing in the initial state between the $q$ and $-q$ modes. Mathematically,

$$
\begin{aligned}
\langle n(q) n(-q)\rangle & =\sinh ^{2} \beta(q) \cosh 2 \beta(q) \neq\langle n(q) n(-q)\rangle_{\mathrm{gG}} \\
& =\langle n(q)\rangle_{\mathrm{gG}}\langle n(-q)\rangle_{\mathrm{gG}}=\sinh ^{4} \beta(q) .
\end{aligned}
$$

As matter of fact, since $n(q) n(-q)$ commutes with $H$, we conclude from the above that $\langle n(q) n(-q)\rangle$ does not relax to the value predicted by $\rho_{\mathrm{gG}}$. Although this defect of $\rho_{\mathrm{gG}}$ can be fixed by enlarging the set of integrals of motion to include the set $I^{\prime}(q)=n(q) n(-q)$ as well, and the corresponding Lagrange multipliers, we shall show below that this is not needed. The reason is that the correlations missing in the generalized Gibbs ensemble as defined above yield a vanishing contribution in the thermodynamic limit to the simplest correlation functions. However, before discussing this point, it is worth mentioning one important exception to the class of observables that relax according to the generalized Gibbs ensemble, namely, the squared fluctuations of the energy

$$
\begin{aligned}
\sigma^{2} & =\left\langle H^{2}\right\rangle-\langle H\rangle^{2} \\
& =\sum_{p, q} \hbar \omega(p) \hbar \omega(q)[\langle n(p) n(q)\rangle-\langle n(p)\rangle\langle n(q)\rangle],
\end{aligned}
$$

which yields $\sigma^{2}=2 \sigma_{\mathrm{gG}}^{2}=\sum_{q} \sinh ^{2} 2 \beta(q) \hbar^{2} \omega(q)^{2}$. Again, since the operator $H^{2}$ is conserved, $\sigma^{2}$ violates the relaxation hypothesis. However, it is tempting to argue since $\sigma^{2}$ (like $\langle H\rangle$ ) is a nonuniversal property of the LM model, this violation is less problematic than a violation in the asymptotic behavior of the correlation functions would be, as the latter tends to be more universal.

In order to proceed with the discussion of the validity of the generalized Gibbs ensemble, let us consider, for the sake of simplicity, the correlations the current operator. In particular, we shall study the following two-time correlation function (no time ordering is implied):

$$
C_{J_{r}}(x, t, \tau)=\left\langle J_{r}(x, t+\tau / 2) J_{r}(0, t-\tau / 2)\right\rangle_{T},
$$

where $\langle\ldots\rangle_{T}$ stands for average over the initial thermal ensemble described by $\rho_{i}=e^{-H_{i} / T} / Z_{0}$, with $H_{i}=H_{0}$ [cf. Eq. (1)]. Using Eqs. (15) and (16), we obtain

$$
\begin{aligned}
C_{J_{r}}(x, t, \tau)= & \frac{1}{(2 \pi)^{2}} \sum_{q>0}\left(\frac{2 \pi q}{L}\right) e^{-q a_{0}} \\
& \times\left\{e^{i q x} f(q, t+\tau / 2) f^{*}(q, t-\tau / 2)\left[1+n_{B}(q, T)\right]\right. \\
& +e^{i q x} g^{*}(q, t+\tau / 2) g(q, t-\tau / 2) n_{B}(q, T) \\
& +e^{-i q x} f^{*}(q, t+\tau / 2) f(q, t-\tau / 2) n_{B}(q, T) \\
& \left.+e^{-i q x} g(q, t+\tau / 2) g^{*}(q, t-\tau / 2)\left[1+n_{B}(q, T)\right]\right\},
\end{aligned}
$$

being $n_{B}(q, T)=\left\langle a^{\dagger}(q) a(q)\right\rangle=\left(e^{-\hbar \omega_{0}(q) / T}-1\right)^{-1} \quad\left(\omega_{0}(q)=v_{F}|q|\right)$ the initial Bose distribution of modes and where $f$ and $g$ are defined in Eqs. (A8) and (A9). In the following, we shall argue that, in the limit $t \rightarrow+\infty$ the above expression reduces to the following correlator in the generalized Gibbs ensemble:

$$
C_{J_{r}}^{\mathrm{gG}}(x, \tau)=\operatorname{Tr}\left[\rho_{\mathrm{gG}}(T) J_{r}(x, \tau) J_{r}(0,0)\right],
$$

where $\rho_{\mathrm{gG}}(T)$ is the extension to an initial thermal state of the generalized Gibbs ensemble introduced above (notice that 
since $\left[H_{f}, I(q)\right]=0$ and therefore $\left[H_{f}, \rho_{\mathrm{gG}}\right]=0$, it is in principle possible to define time-dependent correlation functions on this ensemble, just as they are defined in the equilibrium ensembles). For this (thermal) initial condition (46) fixes the values of $\lambda(q, T)$, which now depend on $\beta(q)$ and on the temperature $T$,

$$
\sinh ^{2} \beta(q)\left[1+n_{B}(q, T)\right]+\cosh ^{2} \beta(q) n_{B}(q, T)=\frac{1}{e^{\lambda(q, T)}-1} .
$$

Introducing this result into the mode expansion for Eq. (52), we arrive at

$$
\begin{aligned}
C_{J_{r}}^{\mathrm{gG}}(x, \tau)= & \frac{1}{(2 \pi)^{2}} \sum_{q>0}\left(\frac{2 \pi q}{L}\right) e^{-q a_{0}\left\{e^{i q(x-v \tau)} \cosh ^{2} \beta(q)[1\right.} \\
& +\langle n(q)\rangle]+e^{i q(x+v \tau)} \sinh ^{2} \beta(q)\langle n(q)\rangle \\
& \left.+e^{-i q(x-v \tau)} \cosh ^{2} \beta(q)\langle n(q)\rangle\right\} \\
& +e^{-i q(x+v \tau)} \sinh ^{2} \beta(q)[1+\langle n(q)\rangle]
\end{aligned}
$$

Using the expressions for $f(q, t)$ and $g(q, t)$ given in Appen$\operatorname{dix}$ A, it can be shown that, in the limit $t \rightarrow+\infty$ of Eq. (51), the rapidly oscillating terms that depend only on $t$ can be dropped and therefore Eqs. (51) and (54) become the same. Thus, the above current correlation function relaxes according to the generalized Gibbs ensemble.

Let us close this section with a digression of higher-order current correlation functions. In principle, these correlations depend on correlations between $n(q)$ and $n(-q)$ (i.e., on $\langle n(q) n(-q)\rangle)$, which exist in the initial state. These are not captured by the above simple-minded generalized Gibbs distribution, which only contains information about the expectation value of $n(q)$ in the initial state. However, a simple argument based on momentum conservation and counting powers of $L$ (the system size) shows that the contribution of these correlations vanishes in the thermodynamic limit, as mentioned above. To illustrate this point, let us consider computing the four-point current correlation function, $\left\langle J_{r}\left(x_{1}, t_{1}\right) J_{r}\left(x_{2}, t_{2}\right) J_{r}\left(x_{3}, t_{3}\right) J_{r}\left(x_{2}, t_{4}\right)\right\rangle_{T}$. Upon using the mode expansion for the current operator $J_{r}(x, t)=\partial \phi_{r}(x, t) / 2 \pi$, the correlation function can be expressed as a sum over expectation values of the mode creation and destruction operators, the $b$ operators. However, it is important that, since each of these mode expansions of the current operator carries a factor of $\frac{1}{\sqrt{L}}$, the four-point correlation function is thus proportional to $L^{-2}$. Upon applying the standard Wick's theorem to the expectation value of the $b$ operators, momentum conservation requires that at least two of the four momenta being summed over must be equal. In general, the two independent momenta are not equal and therefore we get a finite contribution in the limit $L \rightarrow \infty$, which involves only a product of expectation values of the operator $n(q)$. However, when two independent momenta being summed over coincide, the expectation value becomes $\langle n(q) n(-q)\rangle$ and thus, in the thermodynamic limit, the sum turns out to be of order $\frac{1}{L}$, therefore vanishing as $L \rightarrow \infty$. This argument can be extended to higher-order correlations of the current operator. In a sense, it also justifies the use of Wick's theorem when computing higher-order correlations (in the thermodynamic limit) using the above simple-minded generalized Gibbs ensemble.

\section{RELEVANCE TO EXPERIMENTS}

As we described in Sec. I, ultracold atom systems are the ideal arena to study quench dynamics. This is because they are, to a good approximation, isolated systems. Furthermore, as far as one-dimensional systems are concerned, there are already a number of experimental realizations, including experiments where quench dynamics has been already studied $[43,60,61]$. Thus, in this section, we would like to discuss the possible experimental relevance of the results obtained in previous sections. As mentioned above, this must be done with great care because our results have been obtained using a field theory model, namely, the Luttinger model (LM), which can be regarded as "caricature" of the Hamiltonians describing real systems of ultracold atoms confined to one dimension. We must emphasize that the situation in the case of quantum quenches in particular, and of nonequilibrium dynamics in general, is very different from the analysis of low-temperature phenomena in equilibrium. In the latter case, the experimental relevance of models such as the LM is well established by using renormalization-group arguments. This has been put to test over the years using a large variety of numerical and also (when possible) analytical methods. By contrast, in the case of nonequilibrium dynamics, we travel through a largely uncharted land and much needs to be understood in order to achieve a similar level of rigor as in the equilibrium case. Thus, it is convenient to regard models as the LM as "toys," which can provide us valuable lessons and insights into nonequilibrium dynamics of strongly correlated systems. With these cautionary remarks, we can proceed to discuss some experimental systems for which the above results could be of some relevance.

As mentioned in Sec. II, the LM is the exactly solvable model describing the renormalization-group fixed point of a general class of interacting one-dimensional models [55], known as Tomonaga-Luttinger liquids. This class includes systems such as the one-dimensional Bose gas interacting via a Dirac-delta potential (which is solvable via the Bethe ansatz [44]) as well as many other systems of interacting Bose gases with repulsive interactions (such as dipolar) or Fermi gases with both attractive and repulsive interactions. With the caveats of the previous section, it would be interesting to test the results obtained using the LM in one of these systems. However, the dynamics may be strongly modified by the fact that higher-energy states will be also excited following a quantum quench. Such higher-energy states are not, in general, correctly described by the LM. The most unfavorable situation may be expected in the case of a system where interactions are short ranged because, at least from a perturbative point of view, an interaction quench will couple excited states with the same strength. Thus, one possible way around this problem when trying to compare to results for the LM model would be to study experimentally (or numerically) interaction quenches in systems with long-range interactions. This system provides us with a much more faithful realization of the LM, which involves long-range interac- 
tions. Furthermore, the sudden connection of interactions is likely not to scatter particles to high-energy states because interaction decreases with the momentum exchanged between the colliding particles. One system satisfying this requirement is a single-species dipolar 1D Fermi gas confined to one dimension by a strongly anisotropic trapping potential. Since for a single species Fermi gas contact interactions in the $p$-wave channel are weak away from a $p$-wave Feshbach resonance, the dominant interaction is the long-ranged dipolar interaction when the dipoles are all aligned by an external field (electric or magnetic, depending on whether the dipole is electric, such as in heteronuclear molecules, or magnetic, such as in chromium). When confined to a onedimensional trap, the dipolar interaction between the atoms can be approximated by the potential

$$
V_{\mathrm{dip}}(x, \theta)=\frac{1}{4 \pi \epsilon_{0}} \frac{D^{2} \lambda(\theta)}{\left(x^{2}+R_{0}^{2}\right)^{3 / 2}},
$$

where $D$ is the dipolar momentum of the atoms, $\theta$ is the angle subtended by the direction of the atomic motion and the polarizing field, and $\lambda(\theta)=(1-3 \cos \theta)$. Since in this case $g_{2}(q)=g_{4}(q) \propto \lambda(\theta)$, a sudden change in the interactions can be produced by a sudden change in alignment of the field with the direction of motion, that is, a change in $\theta$. In particular, a change in $\theta$ away from the value $\theta_{m}=\cos ^{-1}\left(\frac{1}{3}\right)$ would lead to suddenly switching on the interactions between the fermions [9]. At zero temperature, the momentum distribution $f(p, t)$ (which can be probed by time-of-flight measurements) following the quench into the interacting system would evolve as described in Sec. II B (cf. Fig. 1), with the discontinuity at the Fermi level dying out as $t^{-\gamma^{2}}$. However, currently, atomic gases are produced at temperatures $T \sim 10 \%-20 \%$ of the Fermi energy and this would complicate the observation of this effect. If much lower temperatures could be reached in experiments, so that the application of the LM becomes much more reliable, we expect that in a time of the order of $\hbar / T$, the quenched dipolar gas reaches a stationary state characterized by a momentum distribution that differs from the thermal one. However, the calculations of $f(p, t)$ presented in Sec. II B (cf. Figs. 2 and 3 ) show that the differences between the nonequilibrium and equilibrium results in the stationary state may be well below the current experimental resolution. Alternatively, instead of measuring the momentum distribution, one can try to determine the nonequilibrium exponents by measuring noise correlations in the time-of-flight images [62] or through interferometry [63].

To sum up, we have studied correlations in the LM following an interaction quench. By studying the situations where the interactions are suddenly turned on and off, we have shown that the correlation dynamics is dominated by the initial state correlations at short times. However, in the thermodynamic limit, the LM reaches a nonequilibrium steady state characterized by a set of nonequilibrium exponents that differ from the exponents that describe the decay of the same correlations in equilibrium. This behavior can be obtained from a simple-minded generalized Gibbs ensemble $[9,10,45,46]$. The correlations also exhibit a "light-cone effect," which was previously observed in correlations follow- ing a quantum quench from a gapped to a critical state $[12,15]$. Finally, we have discussed the relevance of our results for the LM for experiments with ultracold atomic gases confined in highly elongated (one-dimensional) traps. We have argued that the most appropriate scenario for the observation of the effects described here may be a onedimensional dipolar Fermi gas.

\section{ACKNOWLEDGMENTS}

We thank T. Giamarchi and A. Muramatsu for useful discussions. A.I. gratefully acknowledges financial support from the Swiss National Science Foundation under MaNEP and Division II, CONICET, UNLP, and hospitality of DIPC, where part of this work was done. M.A.C. thanks M. Ueda for his kind hospitality at the University of Tokyo during his visit at the Ueda ERATO Macroscopic Quantum Control Project of JST (Japan), during which parts of this paper were completed. M.A.C. also gratefully acknowledges financial support of the Spanish MEC through Grant No. FIS200766711-C02-02 and CSIC through Grant No. PIE 200760/007.

\section{APPENDIX A: QUADRATIC HAMILTONIANS}

In this appendix, we study the case of a quantum quench in a model described by a quadratic Hamiltonian

$$
\begin{aligned}
H(t)= & \sum_{q} \hbar\left[\omega_{0}(q)+m(q, t)\right] b^{\dagger}(q) b(q) \\
& +\frac{1}{2} \sum_{q} \hbar g(q, t)\left[b(q) b(-q)+b^{\dagger}(q) b^{\dagger}(-q)\right],
\end{aligned}
$$

where $\left[b(q), b^{\dagger}\left(q^{\prime}\right)\right]=\delta_{q, q^{\prime}}$, commuting otherwise. We will assume that the quench takes place at $t=0$, so that, within the sudden approximation, the system is described by $H_{i}=H(t$ $\leq 0)$ for $t<0$ and by $H_{f}=H(t>0)$ for $t>0$. Furthermore, in order to simplify the analysis, we assume that $m(q, t \leq 0)$ $=g(q, t \leq 0)=0, m(q, t>0)=m(q)$, and $g(q, t)=g(q)$. Notice that the initial Hamiltonian is diagonal in the $b$ operators

$$
H_{i}=H_{0} \equiv \sum_{q} \hbar \omega_{0}(q) b^{\dagger}(q) b(q)
$$

In order to obtain the time evolution of operators $O$ $=\mathcal{O}\left[\left\{b^{\dagger}(q), b(q)\right\}\right]$ after the quench, we recall that, in the Heisenberg picture, $\mathcal{O}(t>0)=\exp \left(i H_{f} t / \hbar\right) \mathcal{O} \exp \left(-i H_{f} t / \hbar\right)$ $=\mathcal{O}\left(\left\{b(q, t), b^{\dagger}(q, t)\right\}\right)$ and therefore all that is needed to solve the above quench problem is to obtain the time evolution of $b(q)$ for $t>0$. For Hamiltonians such as Eq. (A1), this can be done exactly because $H_{f}=H(t>0)$ can be diagonalized by means of the canonical Bogoliubov ("squeezing") transformation

$$
a(q)=\cosh \beta(q) b(q)+\sinh \beta(q) b^{\dagger}(-q) .
$$

Upon choosing

$$
\tanh 2 \beta(q)=\frac{g(q)}{\omega_{0}(q)+m(q)},
$$

the Hamiltonian at $t>0$ is rendered diagonal 


$$
H_{f}=H \equiv E_{0}+\sum_{q} \hbar \omega(q) a^{\dagger}(q) a(q),
$$

where $E_{0}$ is the energy of the ground state of $H$ (relative to the ground-state energy of $H_{0}$ ) and

$$
\omega(q)=\sqrt{\left[\omega_{0}(q)+m(q)\right]^{2}-[g(q)]^{2}}
$$

is the dispersion of the excitations about the ground state of $H_{f}$. The evolution of the $a(q)$ is given by $a(q, t)$ $=\exp \left(i H_{f} t / \hbar\right) a(q) \exp \left(-i H_{f} t / \hbar\right)=e^{-i \omega(q) t} a(q)$. By application of a direct and reverse Bogoliubov transformation, one can obtain the time evolution of $b(q)$,

$$
b(q, t)=f(q, t) b(q)+g^{*}(q, t) b^{\dagger}(-q),
$$

where

$$
\begin{gathered}
f(q, t)=\cos \omega(q) t-i \sin \omega(q) t \cosh 2 \beta(q), \\
g(q, t)=i \sin \omega(q) t \sinh 2 \beta(q) .
\end{gathered}
$$

It is easy to check that Eq. (A7) obeys the initial condition, $b(q, t=0)=b(q)$, and also respects the equal-time commutation rules

$$
\left[b(q, t), b\left(q^{\prime}, t\right)\right]=\left[f(q, t) g^{*}(q, t)-g^{*}(q, t) f(q, t)\right] \delta_{q,-q}=0,
$$

$$
\left[b(q, t), b^{\dagger}\left(q^{\prime}, t\right)\right]=\left(|f(q, t)|^{2}-|g(q, t)|^{2}\right) \delta_{q, q^{\prime}}=\delta_{q, q^{\prime}} .
$$

Thus, a quantum quench described by a quadratic Hamiltonian can be solved by means of a time-dependent canonical transformation.

When the quench is reversed, i.e., when the case with $m(q, t \geq 0)=g(q, t \geq 0)=0$, and $m(q, t<0)=m(q)$ and $g(q, t$ $<0)=g(q)$ is considered, the roles played by the initial and final Hamiltonians are also reversed: the final Hamiltonian is now diagonal in the $b$ 's, $H_{f}=H_{0}$, whereas the transformation of Eq. (A3) renders diagonal the initial Hamiltonian, $H_{i}=H$. Therefore, in this case, the evolution of the $b$ operators is trivial, $H_{f}: b(q, t)=\exp \left[-i \omega_{0}(q) t\right]$, whereas the evolution of the $a$ 's is given by

$$
a(q, t)=f_{0}(q, t) a(q)+g_{0}^{*}(q, t) a^{\dagger}(-q),
$$

where

$$
f_{0}(q, t)=\cos \omega_{0}(q) t-i \sin \omega_{0}(q) t \cosh 2 \beta(q),
$$

$$
g_{0}(q, t)=-i \sin \omega_{0}(q) t \sinh 2 \beta(q)
$$

\section{APPENDIX B: DETAILS OF THE CALCULATION OF THE ONE-PARTICLE DENSITY MATRIX IN THE LUTTINGER MODEL}

In this appendix, we shall provide the details of the calculation of nonequilibrium one-particle density matrix

$$
C_{\psi_{r}}(x, t)=\left\langle\exp \left(i H_{f} t / \hbar\right) \psi_{r}^{\dagger}(x) \psi_{r}(0) \exp \left(-i H_{f} t / \hbar\right)\right\rangle .
$$

To this end, formula (14) is used. In normal ordered form,

$$
\psi_{\alpha}(x)=\frac{\exp \left(-i s_{\alpha} \pi x / L\right)}{\sqrt{L}}: \exp \left[i s_{\alpha} \phi_{\alpha}(x)\right]
$$

where the normal order is defined as

$$
: \exp \left[i s_{\alpha} \phi_{\alpha}(x)\right]:=\exp \left(i \varphi_{\alpha}\right) \exp \left(2 \pi i s_{\alpha} x N_{\alpha}\right) \exp \left[i s_{\alpha} \Phi_{\alpha}^{\dagger}(x)\right] \exp \left[i s_{\alpha} \Phi_{\alpha}(x)\right] .
$$

The boson field $\Phi_{\alpha}(x)$ is given by Eq. (16). Hence,

$$
: \exp \left[-i \phi_{r}(x)\right]: \exp \left[+i \phi_{r}(0)\right]:=\exp \left(-2 \pi i x N_{r} / L\right) \exp \left[\Phi_{r}(x), \Phi_{r}^{\dagger}(0)\right]: \exp \left(-i\left[\phi_{r}(x)-\phi_{r}(0)\right]\right):,
$$

where we have used the identity $e^{A} e^{B}=e^{[A, B]} e^{B} e^{A}$, which holds provided $[A, B]$ is a $c$ number. Using that $\left(a_{0} \rightarrow 0^{+}\right.$is the short-distance cutoff)

$$
\begin{aligned}
{\left[\Phi_{r}(x), \Phi_{r}^{\dagger}(0)\right] } & =\sum_{q>0}\left(\frac{2 \pi}{q L}\right) \exp \left(-q a_{0}\right) \exp (i q x) \\
& =-\ln \left[1-\exp \left(-2 \pi a_{0} / L\right) \exp (2 i \pi x / L)\right],
\end{aligned}
$$

$$
C_{\psi_{r}}(x, t)=G_{r}^{(0)}(x)\left\langle\exp \left[-i F_{r}^{\dagger}(x, t)\right] \exp \left[-i F_{r}(x, t)\right]\right\rangle,
$$

where

$$
G_{r}^{(0)}(x)=\frac{i}{2 L} \frac{1}{\sin \left[\frac{\pi}{L}(x+i a)\right]},
$$

we arrive at the following expression for $C_{\psi_{r}}(x, t)$ :

$$
F_{r}(x, t)=e^{i H t / \hbar}\left[\Phi_{r}(x)-\Phi_{r}(0)\right] \exp \left(-i H_{f} t / \hbar\right)
$$




$$
\begin{aligned}
= & \sum_{q>0}\left(\frac{2 \pi}{q L}\right)^{1 / 2}\left(e^{i q x}-1\right) \\
& \times\left[f(q, t) b(q)+g^{*}(q, t) b^{\dagger}(-q)\right] .
\end{aligned}
$$

To derive the last expression, we have used Eq. (A7). Employing the identities $e^{A} e^{B}=e^{[A, B] / 2} e^{A+B}$ (provided $[A, B]$ is a $c$ number) and that $\left\langle e^{D}\right\rangle=e^{\langle D\rangle+1 / 2\left\langle(D-\langle D\rangle)^{2}\right\rangle}$, we obtain

$$
C_{\psi_{r}}(x, t)=G_{r}^{(0)}(x) \exp \left[-\left\langle F_{r}^{\dagger}(x, t) F_{r}(x, t)\right\rangle\right],
$$

where we have used that $\left\langle\left[F_{r}^{\dagger}(x, t)\right]^{2}\right\rangle=\left\langle F_{r}^{2}(x, t)\right\rangle=0$ because $\left\langle\left[b^{\dagger}(q)\right]^{2}\right\rangle=\left\langle[b(q)]^{2}\right\rangle=\left\langle b^{\dagger}(-q) b(q)\right\rangle=0$, and since the commutator $\left[F_{r}^{\dagger}(x, t), F_{r}(x, t)\right]$ is a $c$ number, it can be safely replaced by $\left\langle\left[F_{r}^{\dagger}(x, t), F_{r}(x, t)\right]\right\rangle$. Note that for $t=0, F_{r}(x, t$ $=0)$ contains only $b(q)$ and thus the average $\left\langle F_{r}^{\dagger}(x, t) F_{r}(x, t)\right\rangle=0$ at $T=0$. In Eq. (B12), the exponent can be expanded to yield

$$
\begin{aligned}
\left\langle F_{r}^{\dagger}(x, t) F_{r}(x, t)\right\rangle= & \sum_{q>0}\left(\frac{2 \pi}{q L}\right) e^{-q a_{0}}\left|e^{i q x}-1\right|^{2}\left[|f(q, t)|^{2} n_{B}(q)\right. \\
& \left.+|g(q, t)|^{2}\left(n_{B}(q)+1\right)\right],
\end{aligned}
$$

being $n_{B}(q)=\left\langle a^{\dagger}(q) a(q)\right\rangle=\left(e^{-\lambda|q|}-1\right)^{-1}$ the distribution of Tomonaga bosons in the initial state (which has been assumed to be a mixed thermal state) and $\lambda=\hbar v_{F} / T$ is the thermal correlation length. We next evaluate explicitly the above result in several limiting cases.

\section{Zero temperature and finite length}

Let us now consider the $T=0$ limit of the above correlator, where $n_{B}(q)=0$, and thus, using Eqs. (A8) and (A9), Eq. (B13) simplifies to

$$
\begin{aligned}
\left\langle F_{r}^{\dagger}(x, t) F_{r}(x, t)\right\rangle_{T=0}= & \sum_{q>0}\left(\frac{2 \pi}{q L}\right) e^{-q a_{0}} \sinh ^{2}[2 \beta(q)](1-\cos q x) \\
& \times[1-\cos 2 v(q)|q| t] .
\end{aligned}
$$

To make further progress, we assume that $\sinh 2 \beta(q)$ $=\gamma e^{-\left|q R_{0}\right| / 2}$, where $R_{0}$ is the range of the interaction. Furthermore, we replace $v(q)$ by $v=v(0),{ }^{6}$ which allows us to safely take the limit $a_{0} \rightarrow 0+$. Next, in order to simplify the computation, we introduce the quantity

$$
\mathcal{E}_{r}(z)=\sum_{q>0}\left(\frac{2 \pi}{q L}\right) \exp \left(-q R_{0}\right) \cos q z,
$$

which can be readily computed to give

$$
\mathcal{E}_{r}(z)=-\ln \left[\frac{\pi}{L} d\left(z+i R_{0} \mid L\right)\right]+\frac{\pi R_{0}}{L}-\ln 2,
$$

where $d(z \mid L)=L|\sin (\pi z / L)| / \pi$ is the cord function. Using this result into Eq. (B14) yields the following expression for the one-particle density matrix:

\footnotetext{
${ }^{6} \mathrm{We}$ assume implicitly that both $\beta(q)$ and $v(q)$ are not singular at $q=0$.
}

$$
\begin{aligned}
C_{\psi_{r}}(x, t> & 0 \mid L)=G_{r}^{(0)}(x \mid L)\left|\frac{d\left(i R_{0} \mid L\right)}{d\left(x+i R_{0} \mid L\right)}\right| \gamma^{2} \\
& \times\left|\frac{d\left(x+2 v t+i R_{0} \mid L\right) d\left(x-2 v t+i R_{0} \mid L\right)}{d\left(2 v t+i R_{0} \mid L\right) d\left(-2 v t+i R_{0} \mid L\right)}\right| \gamma^{2 / 2} .
\end{aligned}
$$

Taking into account that $R_{0} / L \ll 1$, we obtain the result quoted in the main text, Eq. (20), in the scaling limit.

\section{Thermodynamic limit and finite temperature}

We next consider Eq. (B12) for $L \rightarrow \infty$ and finite temperature, $T$. Equation (B13) can be recast as

$$
\left\langle F_{r}^{\dagger}(x, t) F_{r}(x, t)\right\rangle_{T}=\left\langle F_{r}(x, t) F_{r}(x, t)\right\rangle_{T=0}+\mathcal{H}(x)+\mathcal{G}(x, t),
$$

where we have introduced the following functions:

$$
\begin{gathered}
\mathcal{H}(x)=2 \int_{0}^{\infty} \frac{d q}{q} e^{-q a}(1-\cos q x) n_{B}(q), \\
\mathcal{G}(x, t)=2 \gamma^{2} \int_{0}^{\infty} \frac{d q}{q} \exp \left(-q R_{0}\right)(1-\cos q x),
\end{gathered}
$$

$$
[1-\cos (2 v q t)] n_{B}(q)
$$

which hold in the thermodynamic limit and upon replacing $v(q)$ by $v=v(q=0)$ and $\sinh 2 \beta(q)=\gamma \exp \left(-\left|q R_{0}\right| / 2\right)$ as we did in the previous section. We next define the function

$$
g(u ; r)=2 \int_{0}^{+\infty} \frac{d q}{q} e^{-q r} \frac{(1-\cos q u)}{e^{\lambda p}-1},
$$

which can be evaluated to yield [58]

$$
g(u ; r)=2 \ln \left|\frac{\Gamma\left(1+\lambda^{-1} r\right)}{\Gamma\left[1+\lambda^{-1}(r+i u)\right]}\right|,
$$

where $\Gamma(z)$ is the gamma function. In the limit where $r \ll u$ and using that $[58] \Gamma(z) \Gamma(1-z)=\pi / \sin (\pi z)$, the above expression reduces to

$$
g(u ; r)=-\ln \left|\frac{d h(i r \mid T)}{d h(u+i r \mid T)}\right|-\ln \left|\frac{u+i r}{r}\right| .
$$

In the previous expression, we have defined

$$
d h(z \mid T)=\frac{\lambda}{\pi}\left|\sinh \left(\pi \lambda^{-1} z\right)\right| .
$$

Combining this result with Eqs. (B18)-(B21) and (B12), it is seen that the second term in Eq. (B24) exactly cancels the contributions from $G^{(0)}(x \mid L)$ and $\left\langle F_{r}(x, t) F_{r}(x, t)\right\rangle_{T=0}$ in the thermodynamic limit and therefore 


$$
\begin{aligned}
C_{\psi_{r}}(x, t \mid T)= & G_{r}^{(0)}(x \mid T)\left[\frac{d h\left(i R_{0} \mid T\right)}{d h\left(x+i R_{0} \mid T\right)}\right] \gamma^{2} \\
& \times\left[\frac{d h\left(x+2 v t+i R_{0} \mid T\right) d h\left(x-2 v t+i R_{0} \mid T\right)}{d h\left(2 v t+i R_{0} \mid T\right) d h\left(-2 v t+i R_{0} \mid T\right)}\right],
\end{aligned}
$$

where

$$
G_{r}^{(0)}(x \mid T)=\frac{i}{2 \pi} \frac{\pi \lambda^{-1}}{\sinh \left[\pi \lambda^{-1}\left(x+i a_{0}\right)\right]} .
$$

We note that the result of Eq. (B26) can be obtained from Eq. (B17) upon making the replacement $L \sin \left(\pi L^{-1} x\right) / \pi$ by $\lambda \sinh \left(\pi \lambda^{-1} x\right)$. Taking into account that $R_{0} / L \ll 1$, we retrieve the result quoted in the main text, Eq. (34), in the scaling limit.

\section{APPENDIX C: ONE-BODY DENSITY MATRIX OF THE LUTTINGER MODEL IN THE GENERALIZED GIBBS ENSEMBLE}

Next we take up the calculation of the one-body density matrix in the generalized Gibbs ensemble for the Luttinger model discussed in Sec. III. That is, we shall evaluate the expression at $T=0$,

$$
C_{\psi_{r}}^{\mathrm{gG}}(x)=\operatorname{Tr}\left[\rho_{\mathrm{gG}} \psi_{r}^{\dagger}(x) \psi_{r}(0)\right] .
$$

Using the bosonization identity, Eq. (14), we can write the expression as follows:

$$
\begin{aligned}
C_{\psi_{r}}^{\mathrm{gG}}(x) & =G_{r}^{(0)}(x)\left\langle: \exp \left(-i\left[\phi_{r}(x)-\phi_{r}(0)\right]\right):\right\rangle_{\mathrm{gG}} \\
& =G_{r}^{(0)}(x)\left\langle\exp \left[-i \widetilde{F}_{r}^{\dagger}(x)\right] \exp \left[-i \widetilde{F}_{r}(x)\right]\right\rangle_{\mathrm{gG}} .
\end{aligned}
$$

Taking into account that

$$
\begin{aligned}
\tilde{F}_{r}(x)= & \Phi_{r}(x)-\Phi_{r}(0) \\
= & \sum_{q>0}\left(\frac{2 \pi}{q L}\right)^{1 / 2} e^{-q a / 2}\left(e^{i q x}-1\right) \\
& \times\left[\cosh \beta(q) a(q)-\sinh \beta(q) a^{\dagger}(-q)\right] .
\end{aligned}
$$

The expression for $C_{\psi_{r}}^{\mathrm{gG}}(x)$ can be easily computed by using the trick of regarding $\rho_{g G}$ as a canonical ensemble with $q$-dependent temperature. Thus, following the same steps as in the previous section, we arrive at

$$
C_{\psi_{r}}^{\mathrm{gG}}(x)=G_{r}^{(0)}(x) \exp \left[-\left\langle\tilde{F}_{r}^{\dagger}(x) \tilde{F}_{r}(x)\right\rangle_{\mathrm{gG}}\right]
$$

Given that

$$
\left\langle\tilde{F}_{r}^{\dagger}(x) \tilde{F}_{r}(x)\right\rangle_{\mathrm{gG}}=\sinh ^{2} 2 \beta\left[\mathcal{D}_{r}(0)-\mathcal{D}_{r}(x)\right],
$$

where

$$
\begin{aligned}
\mathcal{D}_{r}(x) & =\operatorname{Re}\left\{\sum_{q>0}\left(\frac{2 \pi}{q L}\right) e^{-q R_{0}} e^{i q x}\right\} \\
& =-\ln \left|\sin \frac{\pi}{L}\left(x+i R_{0}\right)\right|-\ln 2-\frac{\pi R_{0}}{L} .
\end{aligned}
$$

Hence, taking the thermodynamic limit

$$
C_{\psi_{r}}^{\mathrm{gG}}(x)=\frac{i}{2 \pi(x+i a)}\left|\frac{R_{0}}{x}\right|^{\gamma^{2}} .
$$

Thus we see that one recovers the same results as $\lim _{t \rightarrow+\infty} C_{\psi_{r}}(x, t)$, Eq. (23).
[1] E. Altman and A. Auerbach, Phys. Rev. Lett. 89, 250404 (2002).

[2] K. Sengupta, S. Powell, and S. Sachdev, Phys. Rev. A 69, 053616 (2004).

[3] R. A. Barankov and L. S. Levitov, Phys. Rev. Lett. 96, 230403 (2006).

[4] E. A. Yuzbashyan, B. L. Altshuler, V. B. Kuznetsov, and V. Z. Enolskii, Phys. Rev. B 72, 220503(R) (2005).

[5] C. Kollath, U. Schollwöck, J. von Delft, and W. Zwerger, Phys. Rev. A 71, 053606 (2005).

[6] E. Altman and A. Vishwanath, Phys. Rev. Lett. 95, 110404 (2005).

[7] A. Ruschhaupt, A. del Campo, and J. G. Muga, Eur. Phys. J. D 40, 399 (2006).

[8] E. A. Yuzbashyan and M. Dzero, Phys. Rev. Lett. 96, 230404 (2006).

[9] M. A. Cazalilla, Phys. Rev. Lett. 97, 156403 (2006).

[10] M. Rigol, V. Dunjko, V. Yurovsky, and M. Olshanii, Phys. Rev. Lett. 98, 050405 (2007).
[11] E. Perfetto, Phys. Rev. B 74, 205123 (2006).

[12] P. Calabrese and J. Cardy, Phys. Rev. Lett. 96, 136801 (2006).

[13] M. Rigol, A. Muramatsu, and M. Olshanii, Phys. Rev. A 74, 053616 (2006).

[14] S. R. Manmana, S. Wessel, R. M. Noack, and A. Muramatsu, Phys. Rev. Lett. 98, 210405 (2007).

[15] P. Calabrese and J. Cardy, J. Stat. Mech.: Theory Exp. 2007, P06008 (2007).

[16] C. Kollath, A. M. Läuchli, and E. Altman, Phys. Rev. Lett. 98, 180601 (2007).

[17] V. Gritsev, E. Demler, M. Lukin, and A. Polkovnikov, Phys. Rev. Lett. 99, 200404 (2007).

[18] M. Eckstein and M. Kollar, Phys. Rev. Lett. 100, 120404 (2008).

[19] M. Kollar and M. Eckstein, Phys. Rev. A 78, 013626 (2008).

[20] M. Moeckel and S. Kehrein, Phys. Rev. Lett. 100, 175702 (2008).

[21] M. Cramer, A. Flesch, I. P. McCulloch, U. Schollwöck, and J. Eisert, Phys. Rev. Lett. 101, 063001 (2008). 
[22] A. Flesch, M. Cramer, I. P. McCulloch, U. Schollwöck, and J. Eisert, Phys. Rev. A 78, 033608 (2008).

[23] M. Rigol, V. Dunjko, and M. Olshanii, Nature (London) 452 , 854 (2008).

[24] S. R. Manmana, S. Wessel, R. M. Noack, and A. Muramatsu, Phys. Rev. B 79, 155104 (2009).

[25] C. De Grandi, R. A. Barankov, and A. Polkovnikov, Phys. Rev. Lett. 101, 230402 (2008).

[26] P. Reimann, Phys. Rev. Lett. 101, 190403 (2008).

[27] A. Faribault, P. Calabrese, and J.-S. Caux, J. Stat. Mech.: Theory Exp. (2009) P03018.

[28] D. Patanè, L. Amico, A. Silva, R. Fazio, and G. E. Santoro, Phys. Rev. B 80, 024302 (2009).

[29] D. Patanè, A. Silva, F. Sols, and L. Amico, Phys. Rev. Lett. 102, 245701 (2009).

[30] D. Rossini, A. Silva, G. Mussardo, and G. Santoro, Phys. Rev. Lett. 102, 127204 (2009).

[31] P. Barmettler, M. Punk, V. Gritsev, E. Demler, and E. Altman, Phys. Rev. Lett. 102, 130603 (2009).

[32] D. Sen, K. Sengupta, and S. Mondal, Phys. Rev. Lett. 101, 016806 (2008).

[33] U. Divakaran, A. Dutta, and D. Sen, Phys. Rev. B 78, 144301 (2008).

[34] S. Mondal, K. Sengupta, and D. Sen, Phys. Rev. B 79, 045128 (2009).

[35] U. Divakaran, V. Mukherjee, A. Dutta, and D. Sen, J. Stat. Mech.: Theory Exp. (2009) P02007.

[36] K. Sengupta, D. Sen, and S. Mondal, Phys. Rev. Lett. 100, 077204 (2008).

[37] V. Mukherjee, U. Divakaran, A. Dutta, and D. Sen, Phys. Rev. B 76, 174303 (2007).

[38] V. Mukherjee, A. Dutta, and D. Sen, Phys. Rev. B 77, 214427 (2008).

[39] G. Roux, Phys. Rev. A 79, 021608(R) (2009).

[40] See, for example, A. Cho, Science 320, 312 (2008).

[41] M. Greiner, O. Mandel, T. Esslinger, T. W. Haënsch, and I.
Bloch, Nature (London) 415, 39 (2002).

[42] M. Greiner, O. Mandel, T. Hänsch, and I. Bloch, Nature (London) 419, 51 (2002).

[43] T. Kinoshita, T. Wenger, and D. S. Weiss, Nature (London) 440, 900 (2006).

[44] E. H. Lieb and W. Liniger, Phys. Rev. 130, 1605 (1963).

[45] E. T. Jaynes, Phys. Rev. 106, 620 (1957).

[46] E. T. Jaynes, Phys. Rev. 108, 171 (1957).

[47] M. Srednicki, Phys. Rev. E 50, 888 (1994).

[48] A. Iucci and M. A. Cazalilla (unpublished).

[49] T. Barthel and U. Schollwöck, Phys. Rev. Lett. 100, 100601 (2008).

[50] J. M. Luttinger, J. Math. Phys. 4, 1154 (1963).

[51] D. C. Mattis and E. H. Lieb, J. Math. Phys. 6, 304 (1965).

[52] S. Tomonaga, Prog. Theor. Phys. 5, 544 (1950).

[53] A. Luther and I. Peschel, Phys. Rev. B 9, 2911 (1974).

[54] F. D. M. Haldane, Phys. Rev. Lett. 45, 1358 (1980).

[55] F. D. M. Haldane, J. Phys. C 14, 2585 (1981).

[56] F. D. M. Haldane, Phys. Rev. Lett. 47, 1840 (1981).

[57] T. Giamarchi, Quantum Physics in One Dimension (Oxford University Press, Oxford, 2004).

[58] A. Gradshteyn and R. Ryzhik, Tables of Integrals Series and Products (Academic Press, New-York, 1980).

[59] R. Balian, From Microphysics to Macrophysics: Applications of Statistical Mechanics (Springer-Verlag, Berlin, 1991), Vol. 1.

[60] K. Günter, T. Stöferle, H. Moritz, M. Köhl, and T. Esslinger, Phys. Rev. Lett. 95, 230401 (2005).

[61] T. Stöferle, H. Moritz, C. Schori, M. Köhl, and T. Esslinger, Phys. Rev. Lett. 92, 130403 (2004).

[62] L. Mathey, E. Altman, and A. Vishwanath, Phys. Rev. Lett. 100, 240401 (2008).

[63] A. Polkovnikov, E. Altman, and E. Demler, Proc. Natl. Acad. Sci. U.S.A. 103, 6125 (2006).

[64] S.-K. Ma, Statistical Mechanics (World Scientific, Singapore, 1985). 